\title{
Enhancement of magnetization plateaus in low-dimensional spin systems
}

\author{
Alexandros Metavitsiadis $\odot,{ }^{1, *}$ Christina Psaroudaki $\odot, 2,3, \uparrow$ and Wolfram Brenig ${ }^{1, \ddagger}$ \\ ${ }^{1}$ Institute for Theoretical Physics, Technical University Braunschweig, D-38106 Braunschweig, Germany \\ ${ }^{2}$ Department of Physics, California Institute of Technology, Pasadena, California 91125, USA \\ ${ }^{3}$ Institute for Theoretical Physics, University of Cologne, D-50937 Cologne, Germany
}

(Received 20 February 2020; accepted 4 June 2020; published 18 June 2020)

\begin{abstract}
We study the low-energy properties and, in particular, the magnetization process of a spin- $1 / 2$ Heisenberg $J_{1}-J_{2}$ sawtooth and frustrated chain (also known as a zigzag ladder) with a spatially modulated $g$ factor. We treat the problem both analytically and numerically while keeping the $J_{2} / J_{1}$ ratio generic. Numerically, we use complete and Lanczos diagonalization as well as the infinite time-evolving block decimation method. Analytically, we employ (non-)Abelian bosonization. Additionally, for the sawtooth chain, we provide an analytical description in terms of flat bands and localized magnons. By considering a specific pattern for the $g$-factor modulation for both models, we show that a small inhomogeneity significantly enhances a magnetization plateau at half saturation. For the magnetization of the frustrated chain, we show the destruction of one-third of the full saturation plateau in favor of the creation of a plateau at half saturation. For large values of the inhomogeneity parameter, the existence of an additional plateau at zero magnetization is possible. Here and at higher magnetic fields, the system is locked in the half-saturation plateau, never reaching full saturation.
\end{abstract}

DOI: $10.1103 /$ PhysRevB.101.235143

\section{INTRODUCTION}

Frustrating interactions in quantum magnets have revealed a plethora of exotic phenomena with no classical analog $[1,2]$. One such example is the appearance of magnetization plateaus, i.e., regions in the magnetization process of a paramagnetic system at which the magnetization stays put at some fractional value $M_{p}$ of the saturation magnetization $M_{s}$ despite the increase of the magnetic field. Magnetization plateaus have been observed in several systems independent of their dimensionality, described by very different geometries, e.g., in Shastry-Sutherland-type models [3-5], triangular [6-9], square [10,11], checkerboard [12,13], and Kagome geometries [14-17], down to one-dimensional (1D) frustrated systems [18-20] and many more (see also Refs. [21-25] for comparative studies). While significant knowledge may have been gathered on the ground state of these systems, the situation often becomes more challenging at the magnetization plateau, where a prerequisite for the existence of a plateau is the opening of a gap in some parts of the spectrum.

Here we use the sawtooth as well as the frustrated chain (see Fig. 1) as prototypical models to investigate the effect of a spatially modulated $g$ factor in systems that exhibit magnetization plateaus. Not only are these models the cornerstones of 1D quantum magnetism but they have also been used to understand physics in higher dimensions. According to the Oshikawa-Yamanaka-Affleck theorem [26,27], a 1D spin- $S$ system with a $p$-periodic ground state could exhibit magne-

\footnotetext{
*a.metavitsiadis@tu-bs.de

†cpsaroud@uni-koeln.de

${ }^{\ddagger}$ w.brenig@ @u-bs.de
}

tization plateaus for values of the magnetization $M$ which satisfy the condition $p S\left(1-M / M_{s}\right) \in \mathbb{Z}$. The sawtooth chain exhibits a magnetization plateau at half saturation $M_{p}=M_{s} / 2$ for a wide range of the ratio $J_{2} / J_{1}$ [28], while the frustrated chain exhibits a magnetization plateau at $M_{s} / 3$ [29].

In this paper, we primarily focus on the sawtooth chain, but we keep the analysis as general as possible to simultaneously treat the frustrated chain and discuss the similarities as well as the differences between the two models. Although the sawtooth chain, as well as variants of it, have been studied theoretically early on [30-44], they remain of great interest today [45-53]. From an experimental point of view, the situation remains challenging, with only a limited number of compounds being reported up to today to materialize dominant magnetic interactions in a sawtooth pattern. Prominent examples are the delafossite $\mathrm{YCuO}_{2.5}$ [54-57], the double spin chain systems $\mathrm{KCuCl}_{3}$ and $\mathrm{TlCuCl}_{3}$ [19], the multiferroic $\mathrm{Mn}_{2} \mathrm{GeO}_{4}$ [58-60], the olivines $\mathrm{Zn}_{2} \mathrm{~S}_{4}(L=\mathrm{Er}, \mathrm{Tm}, \mathrm{Yb})$ [61], and the $\mathrm{Fe}$ chains $\mathrm{Rb}_{2} \mathrm{Fe}_{2} \mathrm{O}\left(\mathrm{AsO}_{4}\right)_{2}$ and $\mathrm{Fe}_{2} \mathrm{O}\left(\mathrm{SeO}_{3}\right)_{2}$ [62,63]. Remarkably, and despite great efforts, a magnetization plateau has not been reported for any of these systems. Very recently, a study on the magnetic structure of the natural mineral atacamite showed that its magnetic structure is that of the sawtooth type with aniferromagnetic couplings between the spin-1/2 moments, with, however, a puzzling magnetization plateau [64].

Our paper is organized as follows. First, in Sec. II, we present the model and its basic properties. In the first part of our results, we present analytical calculations (Sec. III), first in terms of field theory, Secs. III A and III B, and then in terms of localized magnons in Sec. III C. In the second part of our analysis, we present numerical results (Sec. IV) for a uniform or modulated $g$ factor and for both the sawtooth as well as the frustrated chain. We conclude in Sec. V. 


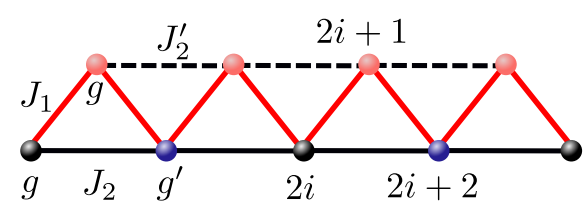

FIG. 1. A generalized 1D chain with anisotropic next-nearestneighbor interactions. The upper base-base coupling is parametrized as $J_{2}^{\prime}=(1-\alpha) J_{2}$, with $\alpha=0,1$. For $\alpha=0\left(J_{2}^{\prime}=J_{2}\right)$, the frustrated chain with NNN interactions, or zigzag ladder, is recovered while for $\alpha=1\left(J_{2}^{\prime}=0\right)$ the sawtooth chain is recovered. The $g$ factor is considered to be either uniform $g^{\prime}=g$ or to vary on every other site of the lower chain with $g^{\prime}=g-\delta g$ and $\delta g>0$, indicated by black and blue colors. For the upper chain, we consider a uniform $g$ value.

\section{MODEL}

Our starting point is the generalized 1D Heisenberg chain with nearest $J_{1}$ neighbor (NN) and anisotropic next-nearestneighbor (NNN) interactions $J_{2}$ and $J_{2}^{\prime}=(1-\alpha) J_{2}$, Fig. 1 . The Hamiltonian that describes this system in the presence of a uniform magnetic field along the $z$-axis $\mathbf{B}=B \hat{z}$ reads

$$
\begin{aligned}
H= & -\sum_{j} g_{j} \mu_{B} B S_{j}^{z}+J_{1} \sum_{j} \mathbf{S}_{j} \cdot \mathbf{S}_{j+1} \\
& +\frac{J_{2}}{1+\alpha} \sum_{j}\left[1+(-1)^{j} \alpha\right] \mathbf{S}_{j} \cdot \mathbf{S}_{j+2},
\end{aligned}
$$

where $\mathbf{S}_{j}$ are spin- $\frac{1}{2}$ operators residing on the lattice sites, $\mu_{B}$ is the Bohr magneton, and we set $\hbar=1$. We consider only two values for the parameter $\alpha=0$, or 1 . The sawtooth chain is recovered for $\alpha=1\left(J_{2}^{\prime}=0\right)$ and the frustrated chain (or zigzag ladder) for $\alpha=0\left(J_{2}^{\prime}=J_{2}\right)$, respectively. Although we are mainly interested in the case of the sawtooth chain, we keep $\alpha$ as a parameter in our analysis to draw analogies between the two models. The ratio of the two couplings $f=$ $J_{2} / J_{1}$ can also be perceived as the degree of frustration. A central point of this paper is our consideration of a particular spatial variation of the $g$ factor. Namely, $g_{j}$ exhibits two patterns: a uniform one $g_{j}=g$ and a modulated one, where the value of the $g$ factor on every second site of the lower chain has a different value $g^{\prime}$, with $g^{\prime}=g-\delta g$ and $\delta g>0$. Note that the sawtooth chain has no leg inversion symmetry and therefore such a modulation only on one part of the system is not unlikely to happen in material realizations, albeit it would probably be relatively weak. On the other hand, tuning the $g$ factor might be an option for, e.g., electric field manipulation on the nanoscale in molecular quantum magnets [65]. Since our focus is the fundamental properties of the model, we consider $\delta g$ to be a free parameter, allowing for values as high as $g$, to provide a complete picture of our theoretical findings (for $\delta g>g$, the $g$ factor exhibits a staggering sign).

The two models, the frustrated chain and the sawtooth chain, share some common properties. They both exhibit either a unique gapless spin fluid [Tomonaga Luttinger liquid (TLL)] ground state or a gapped dimerized one when the degree of frustration is in the range $f_{c_{1}}<f<f_{c_{2}}$, with $f_{c_{1}} \approx$ 0.48 and $f_{c_{2}} \approx 1.53$ for the sawtooth chain and $f_{c_{1}} \approx 0.24$ and $f_{c_{2}}=\infty$ for the frustrated chain (note that the upper critical points $f_{c_{2}}$ are rather challenging due to the exponentially small gaps) [40,66-68]. Both models allow for analytical solutions of their ground states at special values of the frustration ratio ( $f=1$ for the sawtooth chain and $f=1 / 2$ for the frustrated chain) with double degenerate ground states [32,69-71]. The low-lying excitations are kinks and antikinks in the form of domain walls spatially separating regions of one type of ground state. Their dispersion, however, differs with the kink excitations being gapped in the sawtooth and gapless in the frustrated chain [36]. Another difference between the two models appears in the magnetization process of each system. While the sawtooth chain exhibits a plateau at $M_{p}=M_{s} / 2$, the frustrated chain exhibits one at $M_{p}=M_{s} / 3$. For completeness, we mention that the value of the plateau $M_{p}$ depends on the geometrical properties of the model and therefore is independent of the coupling ratio $f$ in contrast to the plateau's width, which depends on the size of the gap in the presence of the magnetic field, and therefore depends on the degree of frustration.

\section{ANALYTICAL RESULTS}

First, we treat the problem analytically by employing Abelian and non-Abelian bosonization focusing on the $J_{1} \gg J_{2}, J_{2}^{\prime}$ regime.

\section{A. Non-Abelian bosonization}

Let us first detour by revisiting the field theory of the sawtooth chain in the absence of a magnetic field in the context of non-Abelian bosonization [72-76]. Within non-Abelian bosonization, both the U(1) and the SU(2) symmetries of the underlying Hubbard model are considered in terms of the bosonic field $\varphi_{c}$ and the matrix field $\mathfrak{g}$. The charge sector is gapped out and the spin operators can be written in terms of chiral $\mathrm{SU}(2)$ currents $\mathbf{J}_{L / R}$ and the staggered magnetization $\mathbf{n}=\operatorname{Tr}(\sigma \mathfrak{g})$, with $\sigma$ Pauli matrices, as

$$
\mathbf{S}(x) \approx \mathbf{J}_{L}(x)+\mathbf{J}_{R}(x)+(-1)^{x} \Omega \mathbf{n}(x),
$$

where the bosonization constant $\Omega$ is of the order of one and it is related to the mass of the charge sector. The field theory is completed by considering one more additional operator, the dimerization $\epsilon$, given by the nonoscillating part of $\epsilon(x) \sim$ $(-1)^{x} \mathbf{S}(x) \cdot \mathbf{S}(x+a) \sim \operatorname{Tr}(\mathfrak{g})[74,77]$.

In the $J_{1} \gg J_{2}, J_{2}^{\prime}$ regime, the system can be considered as a Heisenberg chain with a coupling constant $J_{1}$ perturbed by the couplings $J_{2}, J_{2}^{\prime}$, where each one of the latter couples NNN sites that belong only to one of the two sublattices (the upper or the lower chain). In the continuum, the perturbation of the fixed point Hamiltonian $H_{0}\left(J_{1}\right)$ reads

$$
\delta H=\frac{1}{1+\alpha} \int d x\left[\lambda_{J} \mathbf{J}_{L} \cdot \mathbf{J}_{R}(x)+\lambda_{\partial \epsilon} \partial \epsilon(x)\right] .
$$

The bare couplings $\lambda$ depend on the microscopic couplings $J_{1}, J_{2}, J_{2}^{\prime}$ and the bosonization constant $\Omega$,

$$
\lambda_{J} \sim J^{c}-J_{2}, \quad \lambda_{\partial \epsilon} \sim \alpha \frac{3 \Omega^{2}}{2 \pi} J_{2},
$$

with $J^{c}$ the critical coupling for each model to enter the dimer phase. The current operator is generated by the interaction term of the NN Hamiltonian as well, $\sim \sum S_{j}^{z} S_{j+1}^{z}$, and the NNN couplings modify its bare value. For the frustrated chain 
$(\alpha=0)$, translation symmetry by one site is restored and the Luttinger liquid fixed point is solely disturbed by the current operator, which is known to open a gap at $J_{z z}^{c} / J_{1} \approx 0.241167$ and drive the system in a dimerized phase [67]. On the other hand, for the sawtooth chain $(\alpha=1)$, the strength of the current operator due to the NNN interactions is reduced by a factor of $1 / 2$ while the $\partial \epsilon$ operator appears. The effect of this operator, which is a total derivative, has triggered a big dispute in the literature [39,78-81]. Leaving aside for a moment the $\partial \epsilon$ operator, the operator contents of the two models are identical and the only difference arises in the bare coupling $\lambda_{J}$. This means that the sawtooth chain would undergo a phase transition to a gapped dimerized phase at $J_{s t}^{c}=2 J_{z z}^{c} \approx 0.48$, which coincides remarkably with the value predicted from numerical simulations [40]. In retrospect, one can argue that the $\partial \epsilon$ operator has no effect on the deformation of the critical lines and can, therefore, safely be ignored.

\section{B. Abelian bosonization}

Next, we move to the case of interest, i.e., the sawtooth chain in the presence of a magnetic field with a nonuniform $g$ factor. Here we assume a spatially modulated $g$ factor, which exhibits an alternating pattern taking values $g$ or $g^{\prime}=g-\delta g$ on the lower chain, as described in Fig. 1. This leads to an effective site dependent magnetic field, given by

$$
h(x)=h-\frac{\delta h}{4}\left[1+2 \cos \left(\frac{\pi}{2 a} x\right)+\cos \left(\frac{\pi}{a} x\right)\right],
$$

with $h=g \mu_{B} B$ and $\delta h=\delta g \mu_{B} B$. Therefore, it becomes apparent that the effective magnetic field has a finite overlap not only at momentum $q=0$, but at $q= \pm \frac{\pi}{2 a}$ and $\pm \frac{\pi}{a}$ as well, with the corresponding Fourier components $h_{q}=$ $h-\frac{\delta h}{4}, \frac{\delta h}{4}$, and $\frac{\delta h}{8}$.

Because of the presence of the magnetic field, $\mathrm{SU}(2)$ symmetry is broken, and we turn to Abelian bosonization $[75,76,82]$. The low-energy properties of these systems in the presence of a uniform magnetic field have been described extensively in the literature $[39,44,78,79,83,84]$. Here, we include only what is essential for our work.

In the field theory representation, the spin system is written in terms of chiral fermionic operators which in turn are bosonized in terms of the field $\phi$ and its dual field $\theta$ with $\left[\phi(x), \theta\left(x^{\prime}\right)\right]=-i \vartheta\left(x-x^{\prime}\right)$ and $\vartheta$ the Heaviside step function. This procedure for Hamiltonian Eq. (1) yields the famous TLL Hamiltonian $H_{0}$, perturbed by several operators,

$$
\begin{aligned}
H & =H_{0}+\sum_{j} \int d x \lambda_{j} O_{j}(x), \quad \text { with } \\
H_{0} & =\frac{v}{2} \int d x\left[K[\partial \theta(x)]^{2}+\frac{1}{K}[\partial \phi(x)]^{2}\right],
\end{aligned}
$$

and $v=v\left(m, J_{1}, J_{2}\right), \quad K=K\left(m, J_{1}, J_{2}\right)$ the TomonagaLuttinger parameters $[78,84]$. The perturbative part of the Hamiltonian reads

$$
\begin{aligned}
& O_{1}=\partial \phi, \quad O_{2}^{q}=\cos \left[\beta \phi(x)-\left(2 k_{F}-q\right) x\right], \\
& O_{3}=\cos \left[2 \beta \phi(x)-\left(4 k_{F}-G\right) x-2 k_{F} a\right], \\
& O_{4}=\cos \left[2 \beta \phi(x)-\left(4 k_{F}-G\right) x-4 k_{F} a\right], \\
& O_{5}=\cos \left[2 \beta \phi(x)-\left(4 k_{F}-\pi / a\right) x-4 k_{F} a\right],
\end{aligned}
$$

with

$$
\begin{array}{ll}
\lambda_{1} \sim-\frac{h_{0}}{\sqrt{\pi}}, & \lambda_{2}^{q}=-\frac{h_{q}}{\pi a}, \\
\lambda_{3}=\frac{J_{1}}{2 \pi^{2} a}, & \lambda_{4} \sim \frac{J_{2}}{2 \pi^{2} a(1+\alpha)}, \quad \lambda_{5}=\alpha \lambda_{4} .
\end{array}
$$

Several comments are in order regarding this rich operator content. (i) In Eqs. (6), $\beta$ is a numerical constant, here $\beta=$ $\sqrt{4 \pi}, G$ is the reciprocal lattice vector, which for a system with a unit cell involving $v \geqslant 1$ sites reads $G=\frac{2 \pi}{v a}$, and the Fermi wave vector $k_{F}=\frac{\pi}{2 a}(1-m)$ is given in terms of the magnetization $m=\frac{M}{M_{\text {, }}}$, with $M_{s}=L / 2$. (ii) The operators in Eq. (6a) arise due to the magnetic field whereas the rest from the Heisenberg interactions. (iii) The $O_{2}^{q}$ operator depends on $q$ due to the two Fourier components at $q=\frac{\pi}{2 a}$ and $\frac{\pi}{a}$ of the effective magnetic field. Furthermore, there are two different $\lambda_{2}^{q}$ for each $q$ component. (iv) Not all of these operators survive at every magnetization and/or for any $G$. The rapidly oscillating factors in the arguments of the cosines make them vanish under integration, unless the terms in the parentheses multiplying $x$ vanish. (v) Four-fermion operators yielding in the continuum operators that oscillate with a momentum $q=$ $2 k_{F}[39,85]$ oscillate at the plateau and we drop them here for simplicity. (vi) At finite magnetization, there are additional contributions to $\lambda_{1}$ due to the finite chemical potential in the fermionic representation, which, however, play no role in the discussion of the magentic field dependence.

To determine the behavior of the cosine operators, one needs to consider its behavior under the renormalization group (RG) where the momentum cutoff $\Lambda$ is decreased according to $\delta \Lambda(l)=-\Lambda(l) \delta l$. Assuming the sine-Gordon Hamiltonian $H=H_{0}+g \int d x \cos [\gamma \phi(x)]$, the coupling $g$ of the operator $\cos \gamma \phi$ changes in first order according to $\frac{\delta \ln g}{\delta \ln \Lambda}=$ $d_{\gamma}-2$, with $d_{\gamma}=\frac{K \gamma^{2}}{4 \pi}$ the scaling dimension of this operator. This means that the cosine operator is relevant for $d_{\gamma}<2$, marginal for $d_{\gamma}=2$, and irrelevant for $d_{\gamma}>2$ [75,85]. For example, for vanishing $J_{2}=0$ where $K=1 / 2$, the coefficient in the umklapp term in Eq. (6b) is $\gamma=2 \beta=2 \sqrt{4 \pi}$, i.e., $d_{2 \beta}=2$, namely, the operator is marginal, which agrees with the literature [82].

Let us now discuss the effect of the magnetic field combined with the site modulation of the $g$ factor. The magnetic field contribution is described in Eq. (6a). From there, we see that the strength of the $\partial \phi$ operator is reduced from its uniform value $h$ by $\delta h / 4$. This operator tends to make the field fluctuate, preventing its pinning to some constant value, which in turn would open a gap, and the formation of a magnetization plateau would become possible. In other words, $\partial \phi$ is responsible for destroying magnetization plateaus, and in the presence of the modulation is weakened. The second contribution of the magnetic field comes in the form of the cosine operator in the same equation where the spatial modulation of the $g$ factor yields the $\cos \beta \phi$ term, which is always relevant since $K<2$ [83]. Therefore, since this operator is more relevant than the rest of the operators, it is highly probable to prevail under renormalization and drive the system in a gapped phase, when it is not oscillating. From the above, it becomes apparent that the $g$-factor modulation has a twofold effect on the interaction of the sawtooth with the magnetic 
field. First, it reduces the strength of the operator destabilizing magnetization plateaus and, second, it yields new relevant operators that can stabilize magnetization plateaus.

\section{Sawtooth chain}

We now apply the above to the sawtooth chain $(\alpha=1)$. For a uniform magnetic field $(\delta h=0)$, the lattice periodicity is determined by the two-site unit cell of the microscopic model and, therefore, $G=\pi / a$. The physics at zero magnetization has been described in terms of non-Abelian bosonization in Sec. III. As the uniform magnetic field increases, the $\partial \phi$ operator, which can be absorbed by the substitution $\phi \rightarrow$ $\phi+\frac{\lambda_{1} K}{v} x$, drives the system to an incommensurate phase still described by a TLL fixed point. At some point, a gap opens due to the operators in Eqs. (6b)-(6d) and the system enters the plateau phase. This happens at $m=1 / 2$ where $4 k_{F}=G=$ $\frac{\pi}{a}$ and the oscillating factors in the argument of the cosines vanish [78,83].

By introducing the $g$-factor modulation, the reciprocal lattice vector becomes $G=\frac{\pi}{2 a}$ and the operators $O_{3}$ and $O_{4}$ do not contribute. Hence, there is a competition between the $O_{5}$ and the more relevant $O_{2}^{\pi / 2}\left(d_{\beta}<d_{2 \beta}\right)$, which is expected under RG to first reach the strong coupling limit. The gap of the system scales as $\Delta \sim e^{-\ell}$, where $\ell$ is the point where the perturbative RG breaks down, and the system is no longer conformally invariant [82]. Therefore, since more relevant operators reach the strong coupling limit faster, one could expect a larger gap, meaning a broader magnetization plateau at $m=1 / 2$. This can also be understood from the sine-Gordon model, where the soliton mass relates directly to the plateau width [84] and the soliton mass scales inversely proportionally to the argument of the cosine, at least to leading order in $\gamma$. As a side remark, we mention that the operator $O_{2}^{\pi}$ survives at $k_{F}=\frac{\pi}{2 a}$, albeit with a reduced bare coupling as compared to the coupling of $\mathrm{O}_{2}^{\pi / 2}$, and therefore with fine-tuning of the microscopic parameters a magnetization plateau at $m=0$ may, in principle, arise.

\section{Frustrated chain}

The frustrated chain $(\alpha=0)$ at a uniform field is known to exhibit a magnetization plateau at $m=1 / 3$ due to an even less relevant umklapp operator $[18,29,86]$. However, when the site modulation dependence of the $g$ factor is switched on, the periodicity of the model changes, now $G=\frac{\pi}{2 a}$, and the more relevant operator $O_{2}^{\pi / 2}$ will be present and easily prevail. In fact, the rest of the operators in Eqs. (6) vanish due to the oscillating factors in the argument of the cosine, and it can be safely assumed that the TLL fixed point is solely perturbed by the $O_{2}^{\pi / 2}$ operator. Therefore, a wide plateau is expected at $m=1 / 2$ instead of $m=1 / 3$.

\section{Localized magnons}

We now discuss how the magnetization plateau of the sawtooth chain can be explained in terms of localized magnons that emerge from the frustrated Hamiltonian of Eq. (1) due to a flat energy dispersion relation. Flat dispersions exist for several strongly frustrated spin lattices [24,87], including the
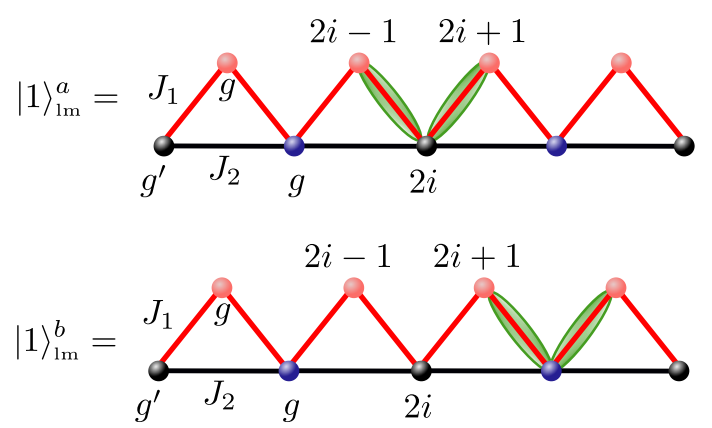

FIG. 2. Localized magnon states realized in the Heisenberg sawtooth chain. The magnon lives on the restricted area indicated by the green ellipses. State $|1\rangle_{\operatorname{lm}}^{a}$ with energy $\varepsilon_{1}^{a}=h-4 J_{2}-(2 / 3) \delta h$ is the lowest eigenstate in the sector $M=L / 2-1$, while state $|1\rangle_{\mathrm{lm}}^{b}$ is a state with higher energy $\varepsilon_{1}^{b}=h-4 J_{2}$.

2D Kagomé lattice [25] and 3D pyrochlore lattice [41], and frustrated electronic systems [88,89].

We first note that in the subspace $M=M_{s}=L / 2$, with $L$ being the total number of spins, the fully polarized state $|F M\rangle$ becomes the ground state for sufficiently large magnetic fields exceeding the saturation field $h_{\text {sat }}$, and plays the role of the vacuum state, $|0\rangle=|F M\rangle$, for the magnon excitations. For $\delta g=0$, the one-magnon state reads

$$
|1\rangle_{k}=\sum_{i=0}^{1} \frac{1}{N_{i}} \sum_{j=1}^{L / 2} e^{i 2 k j} S_{2 j+i}^{-}|0\rangle,
$$

where $S^{-}=S^{x}-i S^{y}, N_{i}$ normalization constants, and $k=$ $4 \pi \frac{l}{L}$, with $l \in \mathbb{Z}$ in the range $\left[0, \frac{L}{2}\right)$. For $f=1 / 2$, it corresponds to a completely flat magnon band $\varepsilon_{1}=h-4 J_{2}$ [28]. A complete flat dispersion suggests that one can construct a localized magnon state in a finite region of the lattice of the form

$$
|1\rangle_{\operatorname{lm}}=l_{2 j}^{\dagger}|0\rangle=\frac{1}{\sqrt{6}}\left(S_{2 j-1}^{-}-2 S_{2 j}^{-}+S_{2 j+1}^{-}\right)|0\rangle,
$$

where the magnon is trapped in a valley indicated by the green ellipses in Fig. 2. Under general assumptions, one can demonstrate that $|1\rangle_{\mathrm{lm}}$ is the lowest eigenstate in the sector $M=M_{s}-1$, and becomes the ground state in an appropriate magnetic field [90,91].

Due to the localized nature of state $|1\rangle_{\mathrm{lm}}$, we proceed to fill the remainder of the lattice with $n$ localized magnons $|n\rangle_{\mathrm{Im}}=l_{2 j}^{\dagger} \ldots l_{2 j^{\prime}}^{\dagger}|0\rangle$, states of lowest energy in the sector $M=M_{s}-n$, with energy $\varepsilon_{n}=n \varepsilon_{1}=n\left(h-4 J_{2}\right)$ above the energy of the ferromagnetic state. To avoid magnon-magnon interactions, magnons are constructed with sufficiently large space separation between them, and $n$ cannot exceed $n_{\max }=$ $L / 4$. We now allow for a finite but small $\delta g>0$. Although states $|n\rangle_{\mathrm{lm}}$ are no longer eigenstates of the Hamiltonian, we can consider $\delta g \ll g$, and calculate their energy within first-order perturbation theory. Two types of localized states can be realized, depending on whether the valley area is centered around a site with $g^{\prime}$ (black) or with $g$ (blue) (see Fig. 2). After a straightforward calculation, we find that states $|n\rangle_{\mathrm{lm}}^{a}$, centered around a site with $g^{\prime}$, have the lowest energy with $\varepsilon_{n}^{a}=n\left(h-4 J_{2}-(2 / 3) \delta h\right)$, while states $|n\rangle_{\mathrm{lm}}^{b}$, centered 
around a site with $g$, remain unaffected by $\delta g$ and have energy equal to $\varepsilon_{n}^{b}=n\left(h-4 J_{1}\right)$. Thus, states $|n\rangle_{\mathrm{lm}}^{a}$ are the lowest energy states in the corresponding sector of magnetization $M$. field,

Under the assumptions specified above, at the saturation

$$
h_{\mathrm{sat}}=\frac{12 g}{3 g-2 \delta g} J_{2} \text {, }
$$

there is a complete degeneracy of all localized-magnon states with energy $\varepsilon_{n}=0$. As a result, $m$ jumps between the saturation value $m=1$ and the value $m=1-n_{\max } / M_{s}=1 / 2$, with $n_{\max }=L / 4$. This is a macroscopic quantum effect and the value of the jump vanishes if the spins become classical. The result above shows that a finite $\delta g$ shifts the saturation field toward larger values, corroborating the field theory prediction for a larger plateau if $\delta g \neq 0$. For higher values of the inhomogeneity parameter $\delta g$, first-order perturbation theory is expected to fail. The full treatment of the problem is involved and is done by means of second-order perturbation theory, taking into consideration the overlap of localized states and propagating states with energy higher than $\varepsilon_{n}$. However, this is beyond the scope of this paper, and we leave it as motivation for future studies.

\section{NUMERICAL RESULTS}

To test the previous theoretical findings, we resort to numerics. We employ two numerical methods, exact diagonalization (ED), namely, full and Lanczos diagonalization, as well as the infinite time-evolving block decimation (iTEBD) method [92,93]. For ED, we use symmetries, total $S_{z}$ conservation, translation by two sites, spin flip for $S_{z}=0$, and parity combined with translation by one site, to reduce the computational effort. In the presence of a uniform magnetic field, the energy levels of the system change according to $E_{n}(h)=E_{n}(0) \pm h M$, where $E_{n}(0)$ is an eigenvalue of the Hamiltonian in the absence of the magnetic field. High-energy states belonging to higher $S_{z}$ sectors will lower their energy in the presence of the magnetic field and will become the ground state as the magnetic field reaches a certain value. This process for a finite system yields finite steps in the magnetization curve, which are not true plateaus but merely finite-size effects. In turn, one needs to discriminate between real magnetization plateaus and finite-size effects.

For a modulated $g$ factor, the situation becomes numerically more demanding. First, the unit cell is enlarged, which creates problems for both methods. Regarding ED, the number of $k$ points is reduced, meaning larger Hilbert spaces for each subsector, creating a memory threshold at smaller system sizes. An additional issue is that the energy levels in the presence of the magnetic field can no longer be evaluated parametrically from the levels without the magnetic field due to the site dependence of $g_{j}$. This means that each value of the magnetic field needs to be evaluated separately, leading to a dramatic increase of the computational time for the larger system sizes. Regarding iTEBD, the $g$-factor modulation causes convergence problems due to the larger unit cell. To avoid this, we rely solely on $\mathrm{ED}$ for $\delta g>0$.

\section{A. Sawtooth chain, uniform $\boldsymbol{g}$ factor}

To correctly interpret the ED results, we first contrast the magnetization under a uniform magnetic field of a sawtooth chain for $f=0.5,1,2$ obtained via ED for $L=28$ spins to that obtained via iTEBD for an infinite system, Fig. 3(a). For all numerical simulations, we assume $h=B$. For the ED results, we plot $M(h)$ as dashed lines, exhibiting finite steps. It has been argued that connecting the middle point of these magnetization steps reproduces the magnetization curve in the thermodynamic limit. In Fig. 3(a), we also show the middle points of the magnetization steps, except at $M_{s} / 2$ where the plateau is expected, and we mark its limiting values. From the agreement of the points to the iTEBD data, one can safely argue that ED gives an excellent qualitative estimate of $M(L \rightarrow \infty)$. The only exception to that is the size of the plateau for $f=2$, which ED tends to overestimate while from the iTEBD it seems rather small. To make this visible, we plot as an inset in panel Fig. 3(a) the magnetization for $f=2$ only in the highlighted region of the main panel. The disagreement between the two methods is attributed to the finite-size behavior of the gap at elevated magnetic fields, which is rather small at this region of the parameter space.

Let us now describe the distinct features of the magnetization curve of the sawtooth chain for each value of frustration $f$. First, for the strongest frustration $f=2$ (weak $J_{1}$ ), we observe a very steep increase of $M$ at low magnetic fields. This reflects the two decoupled-chain limit $J_{1} \rightarrow 0$ where the upper spins, being loosely coupled with the rest of the system, can be very easily polarized. One additional point characteristic of the energy scales is that $M(h)$ is a concave function of the magnetic field before the plateau and a convex function after it. A similar behavior is observed for $f=1$, with a much wider plateau also apparent from the ED data [28]. As the frustration value is further decreased $f=0.5$ (strong $J_{1}$ ), the plateau still extends to a wide range of magnetic field range but the magnetization now displays a convex behavior for $M(h)<M_{s} / 2$, and a concave one for $M(h)>M_{s} / 2$. Hence, the sign of the second derivative of the magnetization $\operatorname{sgn}\left[M^{\prime \prime}(h)\right]$ provides a very useful criterion for the relative strength of the exchange couplings in the system. Decreasing further the degree of frustration (very strong $J_{1}$ ), it would lead to a decrease in the size of the plateau, since the system comes closer to the nonfrustrated Heisenberg chain [94].

In terms of localized magnons for $f=0.5$ (Sec. III C), and using Eq. (10), we find the saturation field to be $h_{\text {sat }} / J=$ $4 J_{2} / J=2.4$, for $J=\left(2 J_{1}+J_{2}\right) / 3$, which is exactly the numerical value obtained from both methods. We also note that at the saturation field, there is a complete degeneracy of all localized-magnon states with energy $\varepsilon_{n}=0$. As a result, $m=M / M_{s}$ jumps between the saturation value $m=1$ and the value $m=1-n_{\max } / M_{s}=1 / 2$, with $n_{\max }=N / 4$.

\section{B. Sawtooth chain, modulated $\boldsymbol{g}$ factor}

Now that the ED has been tested and its results can be correctly interpreted, we will use it to study the magnetization process in the presence of a spatially varying $g$ factor. In Figs. 3(b) and 3(c), we present results for the magnetization in the presence of a modulated factor for different deviations $\delta g$ and for two values of the frustration ratio $f=0.5,2$, 


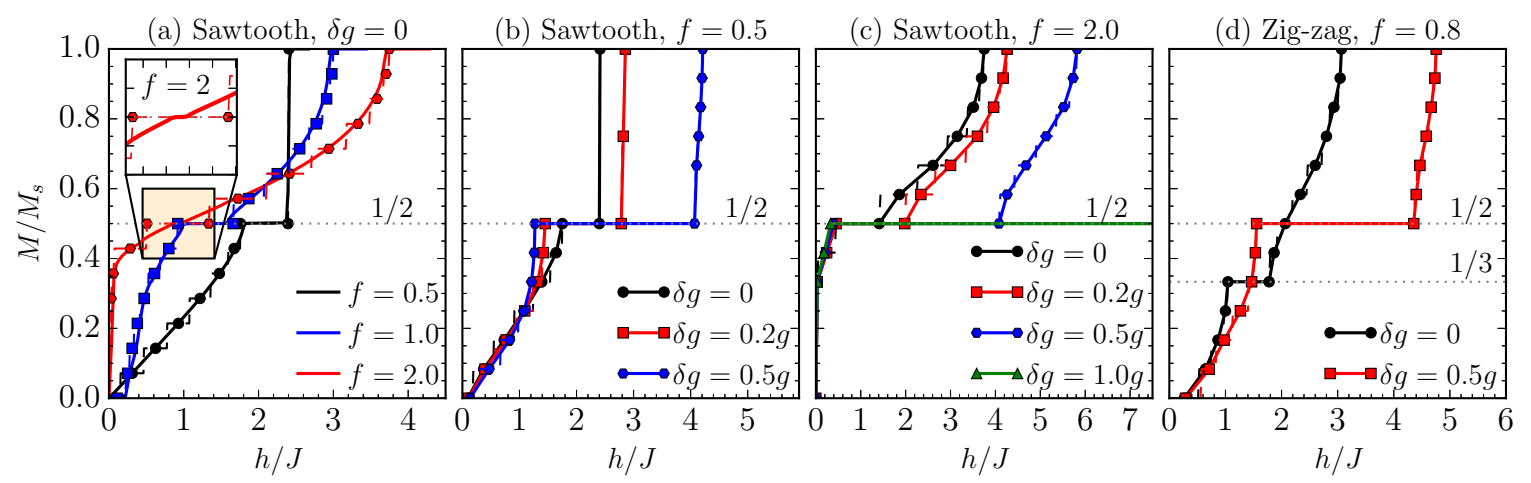

FIG. 3. Magnetization of the sawtooth (a)-(c) and the frustrated (d) chain versus the magnetic field. The magnetic field axis is rescaled by $J=\left(2 J_{1}+J_{2}\right) / 3$ for the sawtooth and by $J=\left(J_{1}+J_{2}\right) / 2$ for the frustrated chain. (a) Comparison of the sawtooth magnetization obtained via ED for $L=28$ sites (thin dashed lines and points) to that obtained via iTEBD (thick solid lines) for three values of the frustration parameter $f=0.5,1,2$ and a uniform $g$ factor, $\delta g=0$. The inset zooms in the highlighted region for $f=2$. (b) Sawtooth magnetization via ED for $L=24$ and $f=0.5$ for three different values of the $g$-factor modulation, $\delta g=0,0.2 g$, $0.5 g$. (c) Sawtooth chain's magnetization via ED for $L=24$ and $f=2$ for four different values of the $g$-factor modulation, $\delta g=0,0.2 g, 0.5 g, g$. (d) Frustrated chain's magnetization via ED for $L=24$ and $f=0.8$ for two different values of the $g$-factor modulation, $\delta g=0,0.5 g$. The points in each panel and for each curve mark the middle of each magnetization step, except when a plateau is expected, where these points mark the beginning and the end of this step.

respectively. We observe that in both cases, a relatively small deviation of $\delta g / g=0.2$ already significantly extends the plateau region. As $\delta g$ is further increased, the plateau grows even more while in the extreme case where $\delta g \geqslant g$, i.e., a $g$ factor with a staggering sign, the system is locked in the half-saturation plateau and never reaches full saturation. Classically thinking, this behavior is to be expected because the spins on the sites which have a $g$ factor of strength $g$ will polarize faster. However, to satisfy the antiferromagnetic interactions of the system, the spins which experience a weaker magnetic field will order antiparallel to the magnetic field to reduce the energy, and therefore the $M_{s} / 2$ plateau is favored. Lastly, we also observe that the $g$-factor modulation introduced here does not affect the sign of $M^{\prime \prime}(h)$, which seems to depend solely on the exchange couplings.

From Sec. III C and Eq. (10), the prediction for the values depicted in Fig. 3(b) is that $h_{\text {sat }} / J=2.8$ for $\delta g=0.2$, which is in remarkably good agreement with the numerical results. For $\delta g=0.5$, the theoretical prediction is $h_{\text {sat }} / J=3.6$, which deviates from the numerical value $h_{\text {sat }} / J=4.1$, suggesting that first-order perturbation theory employed here is insufficient. From Fig. 3(b), it also becomes apparent that for high values of $\delta g$, the degeneracy in $n$ is lifted, and there is a number of critical fields $h_{\text {cr }}(n)$ for which the magnetization $M=M_{s}-n$ changes subsector, with $1 \leqslant n \leqslant n_{\max }$. For the value of $f$ chosen in Fig. 3(c), the picture of localized magnons holds no longer and, therefore, no comparison to the theoretical predictions of Sec. III C can be made.

As far as the prediction of the field theory for a possible plateau at $M=0$ is concerned, if a plateau was to occur at $M=0$, this would happen for weak $J_{2}$ couplings and relatively strong $\delta g$. Comparing Figs. 3(b) and 3(c), we see that the steep increase of $M$ for strong frustration, Fig. 3(c), leaves no room for a possible plateau at $M=0$. In contrast, for weaker values of $f$, e.g., $f=0.5$ [Fig. 3(c)], $M$ develops a smoother onset and with that a finite step at $M=0$. Additional data (not shown here) indicate that for decreasing $f$ and increasing $\delta g$, this first step becomes wider, however, whether or not this could lead to an additional plateau at $M=0$ remains inconclusive.

\section{Zigzag ladder}

Lastly, in the fourth panel of the magnetization data, Fig. 3(d), we apply the same idea but to the zigzag ladder $\left(J_{2}=J_{2}^{\prime}\right)$, which is known to exhibit a plateau at $M_{s} / 3$ for a uniform magnetic field $[18,23]$. Although the zigzag ladder is invariant under chain inversion, the $g$-factor modulation considered here breaks chain reflection symmetry, enlarging the unit cell of the otherwise translationally by-one-site invariant model to four. As one can see in Fig. 3(d), the plateau at $M_{s} / 3$ is destroyed in favor of creating a large plateau at $M_{s} / 2$, as predicted by the field theory calculation.

\section{CONCLUSION}

In conclusion, we presented a comprehensive theoretical study of the $J_{1}-J_{2}$ sawtooth as well as the frustrated chain, focusing on their magnetization process. A unified field theory for both models was developed, and we demonstrated that by introducing a site dependence to the $g$ factor, the magnetization plateau of the sawtooth chain at $M_{s} / 2$ grows for any $J_{2} / J_{1}$ ratio while the $M_{s} / 3$ plateau of the frustrated chain is destroyed in favor of a $M_{s} / 2$ plateau. For a modulation where the $g$ factor vanishes or becomes staggered, we found that the system is locked in the $M_{s} / 2$ plateau, never reaching full saturation. We also emphasized the role of the curvature of $M$ for acquiring an estimate of the microscopic couplings. We anticipate our results will provide guidelines for future theoretical and experimental studies, aiming on new ways to manipulate and extend the plateau region in frustrated magnets, including regimes where the plateau is expected to be small or even nonexisting.

\section{ACKNOWLEDGMENTS}

We are thankful to Leonie Heinze for useful discussions, Xenophon Zotos for his comments on the sine- 
Gordon model, and Stefan Süllow for motivating this work. Work of W.B. has been supported in part by the DFG through Project No. A02 of SFB 1143 (Project No. 247310070), by Nds. QUANOMET, and by the National Science Foundation under Grant No. NSF PHY-1748958.
C.P. has received funding from the European Union's Horizon 2020 research and innovation program under the Marie Sklodowska-Curie Grant Agreement No. 839004. W.B. also acknowledges the kind hospitality of the PSM, Dresden.
[1] L. Balents, Nature 464, 199 (2010).

[2] O. A. Starykh, Rep. Prog. Phys. 78, 052502 (2015).

[3] B. S. Shastry and B. Sutherland, Physica B+C 108, 1069 (1981).

[4] Y. H. Matsuda, N. Abe, S. Takeyama, H. Kageyama, P. Corboz, A. Honecker, S. R. Manmana, G. R. Foltin, K. P. Schmidt, and F. Mila, Phys. Rev. Lett. 111, 137204 (2013).

[5] G. R. Foltin, S. R. Manmana, and K. P. Schmidt, Phys. Rev. B 90, 104404 (2014).

[6] A. V. Chubukov and D. I. Golosov, J. Phys.: Condens. Matter 3, 69 (1991).

[7] A. I. Smirnov, H. Yashiro, S. Kimura, M. Hagiwara, Y. Narumi, K. Kindo, A. Kikkawa, K. Katsumata, A. Y. Shapiro, and L. N. Demianets, Phys. Rev. B 75, 134412 (2007).

[8] Y. Kamiya, L. Ge, T. Hong, Y. Qiu, D. L. Quintero-Castro, Z. Lu, H. B. Cao, M. Matsuda, E. S. Choi, C. D. Batista, M. Mourigal, H. D. Zhou, and J. Ma, Nat. Commun. 9, 2666 (2018).

[9] T. Ono, H. Tanaka, O. Kolomiyets, H. Mitamura, T. Goto, K. Nakajima, A. Oosawa, Y. Koike, K. Kakurai, J. Klenke, P. Smeibidle, and M. Meißner, J. Phys.: Condens. Matter 16, S773 (2004).

[10] K. Morita and N. Shibata, J. Phys. Soc. Jpn. 85, 094708 (2016).

[11] M. E. Zhitomirsky, A. Honecker, and O. A. Petrenko, Phys. Rev. Lett. 85, 3269 (2000).

[12] K. Morita and N. Shibata, Phys. Rev. B 94, 140404(R) (2016).

[13] S. Capponi, Phys. Rev. B 95, 014420 (2017).

[14] S. Nishimoto, N. Shibata, and C. Hotta, Nat. Commun. 4, 2287 (2013).

[15] S. Capponi, O. Derzhko, A. Honecker, A. M. Läuchli, and J. Richter, Phys. Rev. B 88, 144416 (2013).

[16] H. Nakano, Y. Hasegawa, and T. Sakai, J. Phys. Soc. Jpn. 84, 114703 (2015).

[17] T. Picot, M. Ziegler, R. Orús, and D. Poilblanc, Phys. Rev. B 93, 060407(R) (2016).

[18] K. Hida and I. Affleck, J. Phys. Soc. Jpn. 74, 1849 (2005).

[19] W. Shiramura, K.-i. Takatsu, H. Tanaka, K. Kamishima, M. Takahashi, H. Mitamura, and T. Goto, J. Phys. Soc. Jpn. 66, 1900 (1997).

[20] O. A. Petrenko, Low Temp. Phys. 40, 106 (2014).

[21] M. Takigawa and F. Mila, Magnetization plateaus, in Introduction to Frustrated Magnetism: Materials, Experiments, Theory, edited by C. Lacroix, P. Mendels, and F. Mila (Springer, Berlin, 2011), pp. 241-267.

[22] A. Honecker, J. Phys.: Condens. Matter 11, 4697 (1999).

[23] A. Honecker, J. Schulenburg, and J. Richter, J. Phys.: Condens. Matter 16, S749 (2004).

[24] J. Richter, Low Temp. Phys. 31, 695 (2005).

[25] J. Schulenburg, A. Honecker, J. Schnack, J. Richter, and H.-J. Schmidt, Phys. Rev. Lett. 88, 167207 (2002).

[26] M. Oshikawa, M. Yamanaka, and I. Affleck, Phys. Rev. Lett. 78, 1984 (1997).
[27] D. C. Cabra, A. Honecker, and P. Pujol, Phys. Rev. Lett. 79, 5126 (1997).

[28] J. Richter, O. Derzhko, and A. Honecker, Int. J. Mod. Phys. B 22, 4418 (2008).

[29] P. Lecheminant and E. Orignac, Phys. Rev. B 69, 174409 (2004).

[30] T. Hamada, J.-i. Kane, S.-i. Nakagawa, and Y. Natsume, J. Phys. Soc. Jpn. 57, 1891 (1988).

[31] K. Kubo, Phys. Rev. B 48, 10552 (1993).

[32] F. Monti and A. St, Phys. Lett. A 156, 197 (1991).

[33] T. Nakamura and Y. Saika, J. Phys. Soc. Jpn. 64, 695 (1995).

[34] H. Otsuka, Phys. Rev. B 51, 305 (1995).

[35] T. Nakamura and K. Kubo, Phys. Rev. B 53, 6393 (1996).

[36] D. Sen, B. S. Shastry, R. E. Walstedt, and R. Cava, Phys. Rev. B 53, 6401 (1996).

[37] T. Nakamura and S. Takada, Phys. Lett. A 225, 315 (1997).

[38] K. Maisinger and U. Schollwöck, Phys. Rev. Lett. 81, 445 (1998).

[39] S. Chen, H. Büttner, and J. Voit, Phys. Rev. Lett. 87, 087205 (2001).

[40] S. A. Blundell and M. D. Núñez-Regueiro, Eur. Phys. J. B 31, 453 (2003).

[41] J. Richter, J. Schulenburg, A. Honecker, J. Schnack, and H.-J. Schmidt, J. Phys.: Condens. Matter 16, S779 (2004).

[42] M. Nakane, Y. Fukumoto, and A. Oguchi, J. Phys. Soc. Jpn. 75, 114712 (2006).

[43] K. Hida, J. Phys. Soc. Jpn. 77, 044707 (2008).

[44] S. Sarkar and C. D. Hu, Phys. Rev. B 77, 064413 (2008).

[45] S. Bellucci and V. Ohanyan, Eur. Phys. J. B 75, 531 (2010).

[46] Z. Hao, Y. Wan, I. Rousochatzakis, J. Wildeboer, A. Seidel, F. Mila, and O. Tchernyshyov, Phys. Rev. B 84, 094452 (2011).

[47] X. Cai, S. Chen, and Y. Wang, Phys. Rev. A 87, 013607 (2013).

[48] V. Y. Krivnov, D. V. Dmitriev, S. Nishimoto, S.-L. Drechsler, and J. Richter, Phys. Rev. B 90, 014441 (2014).

[49] J.-J. Jiang, Y.-J. Liu, F. Tang, C.-H. Yang, and Y.-B. Sheng, Physica B: Condens. Matter 463, 30 (2015).

[50] D. V. Dmitriev and V. Y. Krivnov, J. Phys.: Condens. Matter 30, 385803 (2018).

[51] A. Baniodeh, N. Magnani, Y. Lan, G. Buth, C. E. Anson, J. Richter, M. Affronte, J. Schnack, and A. K. Powell, npj Quantum Mater. 3, 10 (2018).

[52] S. Paul and A. K. Ghosh, Eur. Phys. J. B 92, 40 (2019).

[53] D. V. Dmitriev, V. Y. Krivnov, J. Schnack, and J. Richter, Phys. Rev. B 101, 054427 (2020).

[54] R. Cava, H. Zandbergen, A. Ramirez, H. Takagi, C. Chen, J. Krajewski, W. Peck, J. Waszczak, G. Meigs, R. Roth, and L. Schneemeyer, J. Solid State Chem. 104, 437 (1993).

[55] S. Capponi, C. Lacroix, O. L. Bacq, A. Pasturel, and M. D. Núñez-Regueiro, J. Phys.: Condens. Matter 19, 145233 (2007).

[56] G. V. Tendeloo, O. Garlea, C. Darie, C. Bougerol-Chaillout, and P. Bordet, J. Solid State Chem. 156, 428 (2001). 
[57] O. Le Bacq, A. Pasturel, C. Lacroix, and M. D. NúñezRegueiro, Phys. Rev. B 71, 014432 (2005).

[58] J. S. White, A. B. Harris, L. C. Chapon, A. Fennell, B. Roessli, O. Zaharko, Y. Murakami, M. Kenzelmann, and T. Kimura, Nat. Commun. 8, 15457 (2017).

[59] T. Honda, Y. Ishiguro, H. Nakamura, Y. Wakabayashi, and T. Kimura, J. Phys. Soc. Jpn. 81, 103703 (2012).

[60] J. S. White, T. Honda, K. Kimura, T. Kimura, C. Niedermayer, O. Zaharko, A. Poole, B. Roessli, and M. Kenzelmann, Phys. Rev. Lett. 108, 077204 (2012).

[61] G. C. Lau, B. G. Ueland, R. S. Freitas, M. L. Dahlberg, P. Schiffer, and R. J. Cava, Phys. Rev. B 73, 012413 (2006).

[62] V. O. Garlea, L. D. Sanjeewa, M. A. McGuire, P. Kumar, D. Sulejmanovic, J. He, and S.-J. Hwu, Phys. Rev. B 89, 014426 (2014).

[63] V. P. Gnezdilov, Y. G. Pashkevich, V. S. Kurnosov, O. V. Zhuravlev, D. Wulferding, P. Lemmens, D. Menzel, E. S. Kozlyakova, A. Y. Akhrorov, E. S. Kuznetsova, P. S. Berdonosov, V. A. Dolgikh, O. S. Volkova, and A. N. Vasiliev, Phys. Rev. B 99, 064413 (2019).

[64] L. Heinze, H. Jeschke, A. Metavitsiadis, M. Reehuis, R. Feyerherm, J. U. Hoffmann, A. U. B. Wolter, X. Ding, V. Zapf, C. C. Moya, F. Weickert, M. Jaime, K. C. Rule, D. Menzel, R. Valentí, W. Brenig, and S. Süllow, arXiv:1904.07820 [condmat.str-el].

[65] F. Troiani, Phys. Rev. B 100, 155424 (2019).

[66] S. R. White and I. Affleck, Phys. Rev. B 54, 9862 (1996).

[67] S. Eggert, Phys. Rev. B 54, R9612 (1996).

[68] M. Kumar, Z. G. Soos, D. Sen, and S. Ramasesha, Phys. Rev. B 81, 104406 (2010).

[69] C. K. Majumdar and D. K. Ghosh, J. Math. Phys. 10, 1388 (1969).

[70] C. K. Majumdar and D. K. Ghosh, J. Math. Phys. 10, 1399 (1969).

[71] W. Caspers and W. Magnus, Phys. Lett. A 88, 103 (1982).

[72] P. Di Francesco, P. Mathieu, and D. Senechal, Conformal Field Theory (Springer, New York, 1997).

[73] I. Affleck, in Fields, Strings and Critical Phenomena, edited by E. Brézin and J. Zinn-Justin, Proceedings of Les Houches
Summer School, 1988 (North-Holland, Amsterdam, 1990), pp. 563-640.

[74] O. A. Starykh, A. Furusaki, and L. Balents, Phys. Rev. B 72, 094416 (2005).

[75] D. Sénéchal, in Theoretical Methods for Strongly Correlated Electrons, edited by D. Sénéchal, A. M. S. Tremblay, and C. Bourbonnais, CRM Series in Mathematical Physics (Springer, New York, 2004), Chap. 4, pp. 139-186.

[76] M. A. Tsvelik, Quantum Field Theory in Condensed Matter Physics (Cambridge University Press, Cambridge, 2003).

[77] A. Metavitsiadis and S. Eggert, Phys. Rev. B 95, 144415 (2017).

[78] S. Sarkar and D. Sen, Phys. Rev. B 65, 172408 (2002).

[79] S. Chen, H. Büttner, and J. Voit, Phys. Rev. B 67, 054412 (2003).

[80] L. Capriotti, F. Becca, S. Sorella, and A. Parola, Phys. Rev. Lett. 89, 149701 (2002).

[81] S. Chen, H. Büttner, and J. Voit, Phys. Rev. Lett. 89, 149702 (2002).

[82] T. Giamarchi, Quantum Physics in One Dimension (Oxford Science Publications, Oxford, 2004).

[83] S. Sarkar, Europhys. Lett. 71, 980 (2005).

[84] K. Totsuka, Phys. Rev. B 57, 3454 (1998).

[85] E. Orignac and T. Giamarchi, Phys. Rev. B 57, 5812 (1998).

[86] O. A. Petrenko, O. Young, D. Brunt, G. Balakrishnan, P. Manuel, D. D. Khalyavin, and C. Ritter, Phys. Rev. B 95, 104442 (2017).

[87] O. Derzhko, J. Richter, and M. Maksymenko, Int. J. Mod. Phys. B 29, 1530007 (2015).

[88] C. Wu, D. Bergman, L. Balents, and S. Das Sarma, Phys. Rev. Lett. 99, 070401 (2007).

[89] D. L. Bergman, C. Wu, and L. Balents, Phys. Rev. B 78, 125104 (2008).

[90] J. Schnack, H.-J. Schmidt, J. Richter, and J. Schulenburg, Eur. Phys. J. B 24, 475 (2001).

[91] H.-J. Schmidt, J. Richter, and R. Moessner, J. Phys. A 39, 10673 (2006).

[92] G. Vidal, Phys. Rev. Lett. 98, 070201 (2007).

[93] R. Orús and G. Vidal, Phys. Rev. B 78, 155117 (2008).

[94] R. B. Griffiths, Phys. Rev. 133, A768 (1964). 